\title{
Perspectives for the allergenicity assessment of key allergens in GM plants
}

\author{
A Fernandez ${ }^{1 *}$, C Mills² $^{2}$, M Lovik ${ }^{3}$, A Spoek ${ }^{4}$, A Germini ${ }^{1}$, A Mikalsen ${ }^{5}$, J-M Wal ${ }^{6}$ \\ From Food Allergy and Anaphylaxis Meeting (FAAM 2013) \\ Nice, France. 7-9 February 2013
}

\section{Background}

According to EU legislation, genetically modified plants (GM plants) should follow a scientific assessment of any risk that they may pose to human and animal health, including an allergenicity assessment. According to the EFSA guidance ${ }^{1}$, the allergenicity assessment of GM plants is structured into the assessment of i) the newly expressed proteins and ii) the whole GM plant. For the latter and as a part of the assessment, if the GM plant receiving the introduced gene(s) is known to be allergenic, its endogenous allergenicity is compared with that of its non-GM comparator(s).

\section{Methods}

Historically, the assessment of endogenous allergenicity has been performed by analytical methodologies based on the use of human sera. Currently, new analytical methods and profiling techniques (e.g. mass spectrometry approaches) not based on human sera are now evolved and could be used for these purposes. In view of these new developments, EFSA recommended the possible inclusion of key allergens in the comparative compositional analysis to limit the use of human sera $[1,2]$.

\section{Results}

There are several limitations associated with the use of human sera, e.g. availability, reproducibility, variability from allergic individuals, difficulty of obtaining wellcharacterised allergic individuals and sera, etc. New technologies, which do not require human sera for the identification and quantification of allergens, have shown to be very useful for risk assessment [3-5]. They may provide important and reliable information on the potential for unintended effects on the endogenous allergenicity (i.e. over expression of allergens) due to the genetic modification.

\section{Conclusion}

In order to avoid the use of human sera, alternative methods based on recent technological advances need to be considered. For the allergenicity assessment of GM plants, the inclusion of key allergens in the comparative compositional analysis, as an additional parameter to be measured by these new methods, could provide robust and reliable information for risk assessment. In this context, when a GM plant is compared with its non-GM comparator(s), an important aspect to be considered is the natural variability and the biological relevance of the identified differences.

\section{Disclosure of interest}

None declared.

\section{Author details}

${ }^{1}$ European Food Safety Authority, Parma, Italy. ${ }^{2}$ Universitiy of Manchester, Manchester, UK. ${ }^{3}$ Norwegian Institute of Public Health, Oslo, Norway. ${ }^{4}$ InterUniversity Research Centre for Technology, Work and Culture, Graz, Austria. ${ }^{5}$ Norwegian Scientific Committee for Food Safety, Oslo, Norway. ${ }^{6}$ INRA-CEA, Gif sur Yvette Cedex, France.

Published: 25 July 2013

\section{References}

1. EFSA: EFSA Journal 2011, 9:2150

2. EFSA: EFSA Journal 2010, 8-1700

Fonseca, et al: J Proteomics 2012, 75:2027-2037.

Houston, et al: J Proteome Res 2011, 10:763-773.

Rouquié, et al: Regulatory Toxicology and Pharmacology 2010, 58:47-53.

doi:10.1186/2045-7022-3-S3-P88

Cite this article as: Fernandez et al:: Perspectives for the allergenicity assessment of key allergens in GM plants. Clinical and Translational Allergy 2013 3(Suppl 3):P88.

${ }^{1}$ European Food Safety Authority, Parma, Italy

Full list of author information is available at the end of the article

(c) 2013 Fernandez et al; licensee BioMed Central Ltd. This is an Open Access article distributed under the terms of the Creative 